\title{
Attenuation of blocking by a change in US locus
}

\author{
KENNETH J. STICKNEY and JOHN W. DONAHOE \\ University of Massachusetts, Amherst, Massachusetts
}

\begin{abstract}
A classical conditioning procedure was instituted in which the locus of the US was changed coincidentally with the onset of the compound conditioning phase of the Kamin blocking design. The nictitating membrane of the rabbit served as the conditioning preparation. Three groups of nine rabbits each were employed: a conventional blocking group in which the US was unchanged during the simple and compound conditioning phases, a control group that received only the compound conditioning phase, and an experimental group given both simple and compound conditioning but in which the US was administered to the contralateral paraorbital region during the compound conditioning phase. Postconditioning presentations of the elements of the compound CS indicated that, relative to the control group, responding during the new element was markedly reduced in the conventional blocking group but was strongly conditioned in the group in which the US locus was changed. Implications of these findings for theoretical analyses of conditioning are discussed.
\end{abstract}

This experiment investigated the effect of a change in the locus of application of the unconditioned stimulus on the behavioral changes produced by the classical procedure. To this end, a variation of the blocking design (Kamin, 1968, 1969) was employed. In the blocking design, a target conditioned stimulus (CS2) is paired with an unconditioned stimulus (US) in simultaneous compound with a previously conditioned stimulus (CS1). When contrasted with the level of responding in control animals not previously trained with CS1, conditioning of CS2 is greatly attenuated, or blocked. The phenomenon of blocking has received extensive experimental corroboration (e.g., Leyland \& Mackintosh, 1978; Marchant \& Moore, 1973) and its theoretical implications are a matter of continuing inquiry (e.g., Mackintosh, 1978; Pearce \& Hall, 1980; Rescorla \& Wagner, 1972).

Regardless of how blocking is ultimately interpreted, the blocking design has shown itself to be a powerful tool for the identification of variables that contribute fundamentally to the conditioning process. First, the existence of blocking indicates that temporal contiguity between CS2 and an otherwise effective US is not sufficient for conditioning: CS2 appears in a favorable temporal relationship with the US, yet control of the CR is attenuated. Second, by changing the parameters of the conditioning procedure coincidentally with the transi-

This research was supported by NIMH Research Grant MH 28610 and a Biomedical Research Support grant to the University of Massachusetts, Amherst. Appreciation is expressed to John W. Moore for the use of his laboratory facilities for the conduct of the work. Requests for reprints should be addressed to John W. Donahoe, Department of Psychology, University of Massachusetts, Amherst, Massachusetts 01003. tion from simple conditioning with $\mathrm{CS} 1$ to compound conditioning with CS1 and CS2, some of the variables that modulate blocking-and, hence, play an important role in conditioning - have been discovered. Among these variables are pre-CS presentations of the US (e.g., Terry, 1976) and various post-CS manipulations of the US (e.g., Dickinson \& Mackintosh, 1978; Donegan, Whitlow, \& Wagner, 1977; Mackintosh, Bygrave, \& Picton, 1977), notably changes in US intensity (e.g., Kamin, 1969; Mackintosh \& Turner, 1971; Wagner, Mazur, Donegan, \& Pfautz, 1980).

The present experiment examined the effect on blocking of another variable, the locus of application of the US. There are several experiments whose outcomes suggest that the locus of the US may affect conditioning in the blocking design. In simple eyelid conditioning with the rabbit, presentation of an additional shock immediately prior to each conditioning trial disrupted conditioning more when the extra shock was given to the same eye to which the US shock was administered than when given to the contralateral eye (Terry, 1976). Similarly, after the galvanic skin response had been conditioned in humans to two CSs paired differentially with US shocks applied to either of two loci on the skin, extinction of the CSs occurred independently (Kimmel \& Reynolds, 1971). Thus, in both of these experiments, the conditioning process was sensitive to the locus of application of the US.

To investigate the contribution of US locus to conditioning in the blocking design, the nictitating membrane (NM) preparation of the rabbit was used (Gormezano, 1966). In this preparation, a CS is paired with an electric shock administered to the 
paraorbital region of one eye with the result that the CS comes to control the shock-elicited movement of the NM in that eye. In the present experiment, the site of application of the US was switched to the paraorbital region of the contralateral eye upon the transition from the simple to the compound conditioning phase of the blocking design. Other work with the NM preparation encouraged the conjecture that the two membranes might be independently conditionable (e.g., Kettlewell, O'Connell, \& Berger, 1974; Salafia, Daston, Bartosiak, Hurley, \& Martino, 1974).

\section{METHOD}

\section{Subjects}

Twenty-seven experimentally naive New Zealand rabbits, obtained from a local supplier, served as subjects.

\section{Apparatus}

The apparatus and techniques used in the conditioning of the NM response were similar to those developed by Gormezano (1966). For all animals, the NM responses of each eye were monitored by minitorque potentiometers (Conrac Model 85153) mounted on each side of the plastic device that restrained bodily movement. Head movement was eliminated by a modification of the basic technique in which bolts, secured to the skull of the rabbit by dental cement, were inserted in a horizontal plate attached to the restraint device. When the nuts placed on these bolts were tightened down to the plate, head movement was prevented.

Each NM was attached to its potentiometer via a nylon loop sutured through the membrane. A metal hook was inserted in the loop to connect the membrane to the lever arm of the potentiometer. The voltage changes across the potentiometer were monitored by a Grass Model D oscillograph. A criterion response was defined as an upward deflection greater than $1 \mathrm{~mm}$ of the oscillograph pen, a deflection that corresponded to a 1-mm movement of the NM.

CS1 was a $1,200-\mathrm{Hz}$ tone at an intensity of $76 \mathrm{~dB}\left(\mathrm{re} 20 \mu \mathrm{N} / \mathrm{m}^{2}\right.$ ) and a duration of $500 \mathrm{msec}$ presented by a speaker centrally mounted in front of the rabbit. CS2 consisted of the illumination for $500 \mathrm{msec}$ of two flashing $(10-\mathrm{Hz}) 6-\mathrm{V}$ dc lights mounted behind translucent white panels on each side of the speaker. The US was a 1-mA ac shock of 50-msec duration applied across a pair of wound-clip electrodes affixed to one or the other paraorbital region of the rabbit. An intensity of $1 \mathrm{~mA}$ was selected following preliminary work that indicated that a shock of this intensity was sufficient to support conditioning without eliciting significant movement in the contralateral NM. Two rabbits were run simultaneously within separate drawers of a ventilated, sound-attenuating file cabinet.

\section{Procedure}

Prior to the conditioning experiment, all animals underwent surgery in which three bolts were secured to the skull by threading three widely spaced jeweler's screws partially into the skull, covering these screws with dental cement, and then embedding the heads of the bolts in the cement until it hardened. After a 5-day recovery period, sutures were placed in both NMs, pairs of wound clips were affixed to both paraorbital regions, and the rabbits were given two 50 -min habituation sessions in the restraint device within the conditioning chambers.

The animals were randomly assigned to one of three equalsized groups. A control group was given compound conditioning (CS1 and CS2). A blocking group was given simple conditioning, with CS1 followed by compound conditioning. Lastly, a switch group was given simple conditioning followed by compound conditioning in which the locus of the US was switched to the contralateral paraorbital region coincidentally with the beginning of compound training.

The procedures for the three groups were implemented as follows. During simple conditioning (Phase 1), the animals in the blocking and switch groups received daily sessions of CS1-US pairings in which the offset of the two stimuli was coterminous. For the blocking group, the US was administered to the right paraorbital region; for the switch group, the US was administered to the left paraorbital region. Each session consisted of 100 trials separated by a variable intertrial interval averaging $30 \mathrm{sec}$. Phase 1 continued until each rabbit in the blocking and switch Groups had achieved an average of $90 \%$ or greater CRs over 4 consecutive days of training. During Phase 1 , animals in the control group were restrained in the experimental chamber and neither CS1 nor the US was presented. The animals were run in four squads of six rabbits and one squad of three rabbits. The rabbits in the control group received a number of habituation sessions equal to the number of sessions required by the slowest animal within the squad to reach the conditioning criterion.

During compound conditioning (Phase 2), the animals in all three groups were treated identically. A compound stimulus consisting of CS1 and CS2 was paired with a US delivered to the right paraorbital region. Five daily sessions of compound conditioning were administered to all animals. Note that the application of the US to the right paraorbital region represented a change in US locus for animals in the switch group only.

Control over the $C R$ by the components of the compound CS was evaluated during a final, test phase in which CS1 and CS2 were presented separately with the US omitted. Two test sessions were employed, each consisting of 50 presentations of CS1 and CS2 at the same intertrial intervals used in the conditioning sessions.

Throughout all conditioning and test sessions, both membranes of all animals were connected to potentiometers and both pairs of wound-clip electrodes were attached to shock wires. Movement of the NM was recorded whenever stimuli were presented.

\section{RESULTS}

The findings are successively described for Phase 1 of simple conditioning, Phase 2 of compound conditioning, and the test phase. Within each phase, responding in the shocked eye is reported first and responding in the unshocked eye second.

\section{Phase 1}

The conditioning criterion-four successive sessions over which NM responses in the shocked eye exceeded an average of $90 \%$ - was met by all animals. The criterion response percentage during CS1 was met in 8.2 sessions by the blocking group and in 9.0 sessions by the switch group. During the criterion sessions, the average latency of the NM response was $317 \mathrm{msec}$ in the blocking group and $320 \mathrm{msec}$ in the switch group. No reliable differences in either sessions to criterion or CR latency were found between the two groups $[\operatorname{Fs}(1,16)=.18$ and 1.51 respectively, $\mathrm{p}>.05$ in both cases]. Thus, conditioning to CS1 proceeded similarly whether shock was administered to the right or left paraorbital regions. 
Responding in the unshocked eye remained at low levels throughout Phase 1. As measured during the last session of training, NM responses averaged $12.2 \%$ and $5.8 \%$ of CS1 presentations for the blocking and switch groups, respectively. Two animals in each group showed no criterion responses in the unshocked eye. For those few responses that did occur, the average latencies were $415 \mathrm{msec}$ for the blocking group and $\mathbf{4 0 4} \mathrm{msec}$ for the switch group. A statistical test indicated that, for those animals that responded in both eyes, the latency of NM responses was greater in the unshocked than in the shocked eye $[F(1,12)=11.9, p<.05]$. An examination of the amplitudes of responses further substantiated the distinction between NM responses in the shocked and unshocked eyes: in all cases, the amplitudes of the responses in the shocked eye exceeded those of the unshocked eye.

\section{Phase 2}

The course of responding in the shocked eye following the institution of compound conditioning is shown in the upper panel of Figure 1. Clearly, the percentage of $\mathrm{NM}$ responses differed among groups at the outset of conditioning with the CS1CS2 compound. In the blocking group, for which the US locus remained unchanged from Phase 1, the level of responding remained in excess of $90 \%$. In the switch group, the percentage of NM responses fell during the first session of compound conditioning and rapidly rose above $90 \%$ thereafter. The control group, which had not received prior conditioning trials in Phase 1, displayed an initially low level of responding but required only three sessions before NM responding was above $90 \%$. Statistical tests confirmed these different trends during compound conditioning. The three groups differed in the level of responding as a function of conditioning sessions $[F(8,96)=16.5, p<.05]$. Orthogonal partitions of the interaction indicated that the blocking group, which showed no deficit, differed from the other groups as a set $[F(4,96)=17.4, p<.05]$ and that the control group, which first began conditioning in Phase 2, differed from the switch group $[\mathrm{F}(4,96)=15.6, \mathrm{p}<.05]$.

At the completion of five sessions of compound conditioning, all groups were responding in the shocked eye to over $90 \%$ of the presentations of the compound CS with an average latency of $321 \mathrm{msec}$. Neither the percentage nor the latency of NM responding differed among the three groups ( $F<1$ for both measures). Thus, prior to the test phase, responding in the shocked eye was comparable for the blocking, switch, and control groups.

The course of responding in the unshocked eye is shown in the lower panel of Figure 1. For animals in the switch group, which had previously received

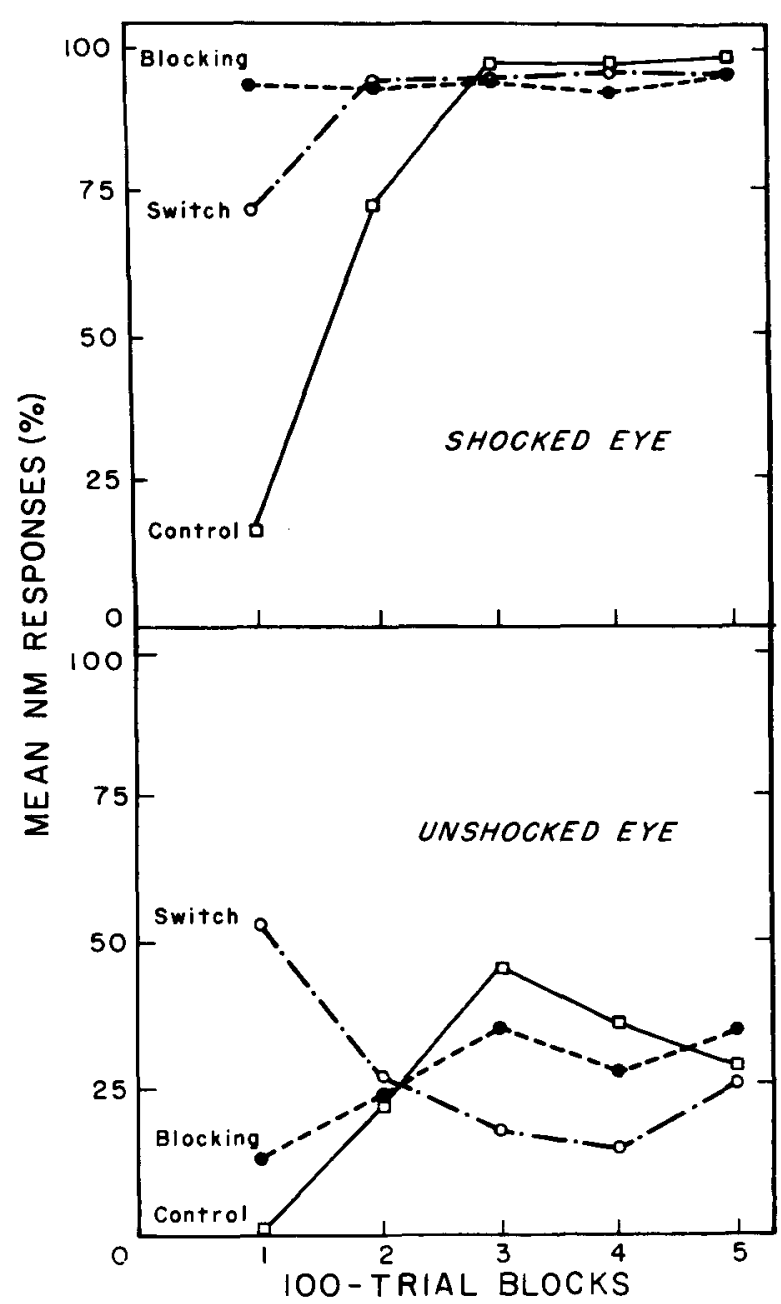

Figure 1. Mean percentage of $N M$ responses as a function of trial blocks during compound conditioning (Phase 2) for the control, switch, and blocking groups. The upper panel displays NM responding from the eye receiving paraorbital shock; the lower panel displays concurrent responding from the unshocked eye.

shock to the now unshocked eye, NM responding was initially frequent and then declined as the shock US was applied to the contralateral eye. For the blocking and control groups, responding in the unshocked eye remained low throughout compound conditioning, although it increased slightly over sessions. The different trends observed in NM responding in the unshocked eye were statistically reliable. The course of responding differed among the three groups $[F(8,96)=5.55, p<.05]$, with orthogonal partitions revealing that the downward trend of the switch group differed from the combined trend of the other two groups $[F(4,96)=10.6, p<.05]$ and that the trends of the blocking and control groups did not differ from one another $(F<1)$.

After five sessions of compound conditioning, 
NM responding in the unshocked eye was at a level of $29 \%$, with an average latency of $368 \mathrm{msec}$ across all three groups. There were no differences among groups in either response frequency or latency $(F<1$ for both measures). The latencies of responses in the unshocked eye were significantly longer than those in the shocked eye across all groups $[F(1,19)$ $=30.9, \mathrm{p}<.05]$. During the final session of compound conditioning, NM responding was totally absent from the unshocked eye of two subjects in the switch group, two subjects in the blocking group, and one subject in the control group. Further evidence for the independence of NM responding in the two eyes was provided by contingency tables for the joint presence and absence of CRs in the shocked and unshocked eyes of the switch group. Contingency tables were computed separately for each subject to avoid potential distortions produced by summing over subjects (Hintzman, 1980). Of the 27 possible such tables during the last three sessions of training when the nine subjects in the switch group were responding at asymptotic levels in the shocked eye, only two tables departed reliably ( $p<$ .05 ) from the joint frequencies expected on the basis of independent responding of the two membranes. Thus, the proportion of statistically significant outcomes $(2 / 27=.07)$ was approximately equal to the expected value of the Type I error rate.

\section{Test Phase}

Following the attainment by all groups of comparably high levels of responding in the shocked eye and comparably low levels in the unshocked eye, conditioning to the elements of the compound CS was separately assessed during extinction. As measured by the percentage of CRs on test trials (see Figure 2, upper panel), responding during CS1 and CS2 was more nearly equal for the control and switch groups than for the blocking group. The differential pattern of responding was statistically significant. The interaction of stimulus elements with groups was reliable $[F(2,24)=4.41, p<.05]$ and, when orthogonally partitioned, was composed of a significant comparison between the blocking group and the combined control and switch groups $[F(1,24)$ $=8.27, \mathrm{p}<.05$ ] and a nonsignificant comparison between the control and switch groups $(F<1)$. Thus, the blocking group varied from the control and switch groups as a set with respect to the difference in responding to CS1 and CS2, while the control and switch groups did not vary in this respect.

A second measure of differential responding to the stimulus elements was obtained by expressing the number of responses during CS2 as a proportion of the total number of responses during extinction. Since the number of responses during CS1 did not vary reliably among the three groups $(F<1)$ but responding during $\mathrm{CS} 2$ did $[F(2,24)=5.04, \mathrm{p}<$
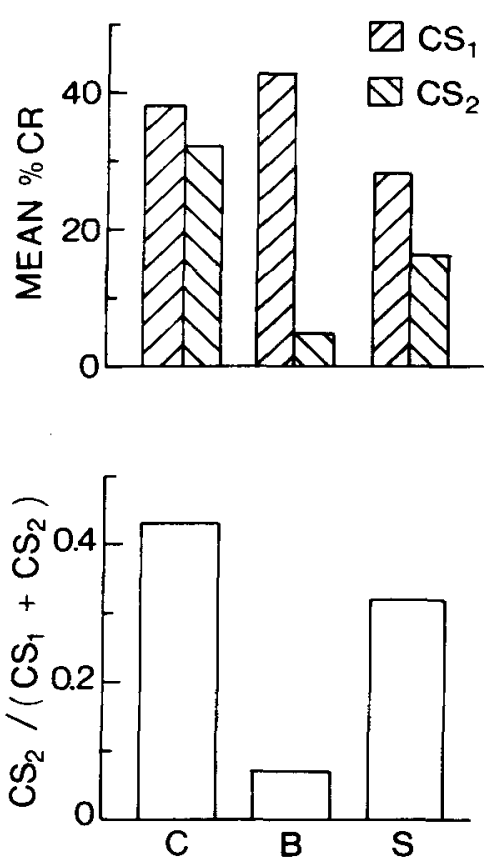

Figure 2. Conditioned responding during the test phase as measured by the percentage of NM responses during CS1 and CS2 (upper panel) and the proportion of the total responses during CS2 (lower panel). The measures are shown separately for the control (C), blocking (B), and switch (S) groups.

$.05]$, the proportion of CS2 responses represents a measure of CS2 responding that controls for individual differences in the absolute level of responding during extinction. A proportion of .5 represents equal responding during $\mathrm{CS} 1$ and $\mathrm{CS} 2$; a proportion of .0 represents no responding during CS2 (i.e., complete blocking of stimulus control by CS2). As shown in the lower panel of Figure 2, the proportion of responses during CS2 was much lower in the blocking group than in the control and switch groups. The differences among the three groups were statistically reliable $[F(2,24)=11.1, p<.05]$. Orthogonal partitions of the group effect indicated that the blocking group differed reliably from the control and switch groups as a set $[F(1,24)=20.2$, $\mathrm{p}<.05]$ and that the control and switch groups did not differ reliably from one another $[F(1,24)=1.91$, $\mathrm{p}>.05]$.

To summarize, both measures of responding in the test phase indicated that conditioning during CS2 was reduced in the blocking group relative to that in the control and switch groups. Thus, the blocking phenomenon was replicated. With respect to the central issue of the effect of a change in US locus on blocking, neither measure indicated a reliable difference between the switch and control groups. Thus, a change in US locus clearly attenuated blocking.

During the test phase, NM responses in the eye 
not shocked during the previous compound conditioning phase were infrequent, occurring on only $\mathbf{9 . 0 \%}$ of the stimulus presentations averaged across groups. The proportion of responses occurring during CS2 was $43 \%$, indicating approximately equal control by the two components of the compound CS. No reliable group differences were found in the proportion of NM responses in the previously unshocked eye $[F(2,24)=2.25, p>.05]$.

\section{DISCUSSION}

In the current experiment, the new element of a compound conditioned stimulus became an effective CS when the locus of the US was changed between the simple and compound conditioning phases of the blocking design. Thus, with a procedure that was otherwise sufficient to produce blocking, changing the locus of the US permitted the acquisition of control by a stimulus in simultaneous compound with a previously conditioned element.

The potent effect of a change in US locus on conditioning is amenable to a number of interpretations. On a behavioral level, a change in US locus alters the NM response elicited by the US and thereby increases the discrepancy between CS-elicited and US-elicited responses present at the outset of the compound conditioning phase. With an invariant locus of shock, however, the corresponding discrepancy between CS- and US-elicited responses is minimal. If the magnitude of the discrepancy between conditioned and unconditioned responding is fundamental to the acquisition of stimulus control, then conditioning of the new stimulus element should be enhanced in the switch group relative to the blocking group (Donahoe, Crowley, Millard, \& Stickney, 1982; Rescorla, 1969). Alternatively, changing the US locus may have elicited a more vigorous UR than would have been obtained with an invariant US locus. A changed US locus could reasonably be assumed to produce less habituation of the UR, a result that would also increase the discrepancy between CS- and US-elicited responses in the switch group relative to the blocking group. Prior presentations of US alone at an invariant locus are known to reduce subsequent conditioning in the NM preparation (Mis \& Moore, 1973; Suboski, DiLollo, \& Gormezano, 1964).

A number of interpretations that appeal to inferred processes (Donahoe \& Wessells, 1980, pp. 6264) postulated to underlie the observed behavioral processes are also possible. From the perspective that conditioning consists of the formation of associations, acquisition occurs when there is a discrepancy between the asymptotic association value supportable by a US and the total association value of all contemporaneous stimuli (Rescorla \& Wagner,
1972). In these terms, the present results could be interpreted to mean that different US loci give rise to at least partially independent asymptotic association values (cf. Bakal, Johnson, \& Rescorla, 1974; Moore \& Stickney, 1980; Rescorla, 1980). From the perspective that conditioning leads to the formation of cognitions, conditioning occurs when the putative CS is followed by a surprising or unexpected US (e.g., Dickinson \& Mackintosh, 1979; Kamin, 1968). In these terms, the present results indicate that the expectation of the US includes a representation of the locus of the US in addition to such properties as US intensity. Finally, from an information-processing perspective, conditioning occurs when a US that is not already represented, or "primed," in short-term memory (Wagner, 1981 ) is presented in these terms, a change in US locus may be interpreted as causing memory to enter an activated state of rehearsal, since prior conditioning has only primed a representation of the US at the original locus. A theme common to all inferredprocess accounts-whether they are stated in terms of associations, expectancies, or memories-is that some representation of US locus is required. All interpretations suggest a more elaborated characterization of the functional relationship between the CS and CR produced by the classical procedure (cf. Pearce, Montgomery, \& Dickinson, 1981).

Within the present experiment, there is evidenceother than the attenuation of blocking by a change in US locus-that the control of responding in the two NMs involved somewhat independent processes. In the compound conditioning phase with the switch group, when substantial responding was occurring with both NMs, responding was being acquired in the newly shocked eye while simultaneously being extinguished in the previously shocked eye. Moreover, when the interrelationships were examined between responding in the two eyes, the presence or absence of NM responses in one eye was not predictive of the occurrence of NM responses in the other eye.

Although the foregoing results indicate that responding within individual trials occurred relatively independently in the two NMs, responding in the newly shocked eye was acquired more rapidly in the switch group during compound conditioning than during original acquisition of simple conditioning. Two origins of this facilitation of the $a b$ solute level of NM responding may be identified. First, acquisition of the NM response is known to require a three-state model to describe the conditioning process accurately (Theios \& Brelsford, 1965; cf. Prokasy \& Gormezano, 1979). These three states may be characterized as an initial nonconditioned state, an intermediate aroused or sensitized state, and a terminal conditioned state. In these terms, 
the facilitative effect in the switch group may be due to a nonspecific sensitization produced by prior contralateral US presentations. A second source of the facilitative effect probably arises from the greater net salience of the compound CS than the simple CS (Rescorla \& Wagner, 1972). Consistent with this interpretation, compound conditioning in the control group proceeded more rapidly than original conditioning with the simple CS in the blocking and switch groups. In summary, the absolute level of responding may be influenced by nonspecific factors such as sensitization or salience, but the occurrence of NM responses on individual trials appears to be due to at least partially independent conditioning processes.

Regardless of how the effects of a change in US locus on conditioning are ultimately to be interpreted, a more extensive experimental analysis of the phenomenon appears to be warranted. When the locus of the US is changed, both the affectors stimulated by the US and the effectors activated by the US are altered. The separable contributions of these factors have yet to be isolated and determined. By so doing, we will come closer to answering the fundamental question posed by Kamin in one of the earliest papers on blocking: "What are the necessary and sufficient conditions for the establishment of an association between CS and US within a Pavlovian paradigm?" (Kamin, 1969, p. 63). Even at this relatively late date, basic questions concerning the empirical foundation of conditioning (cf. Rozeboom, 1958) remain. The answer to Kamin's question is all the more important because recent theoretical work suggests that a single, unified reinforcement principle may accommodate conditioning within both the classical and operant paradigms (Donahoe et al., 1982; Lajoie \& Bindra, 1976).

\section{REFERENCE NOTE}

1. Theios, J., \& Brelsford, J., Jr. A Markov model for classical conditioning: Applications to eye-blink conditioning in rabbits (Tech. Rep 9.) Austin, Tex: University of Texas, Conditioning Research Laboratory, Department of Psychology, 1965.

\section{RWFERENCES}

Baxal, R., Johnson, R., \& Rescorla, R. A. The effect of changing the quality of the reinforcer on blocking. The Pavlovian Journal, 1974, 2, 97-103.

Dickinson, A., \& Mackintosh, N. J. Classical conditioning in animals. Annual Review of Psychology, 1978, 21, 587-612.

Dickinson, A., \& Mackintosh, N. J. Reinforcer specificity in the enhancement of conditioning by posttrial surprise. Journal of Experimental Psychology: Animal Behavior Processes, $1979,5,162-177$.

Donahoe, J. W., Crowley, M. A., Millard, W. J., \& Stickney, K. J. A unified reinforcement principle: Some implications for matching. In M. L. Commons, R. J. Herrnstein, \& H. Rachlin (Eds.), Quantitative analysis of behavior (Vol. 2): Matching and maximizing accounts. Cambridge, Mass: Ballinger, 1982.
Donahoe, J. R., \& Wessells, M. G. Learning, language, and memory. New York: Harper \& Row, 1980.

Doneann, N., Whitlow, J. W., \& Wagner, A. R. Post-trial reinstatement of the CS in Pavlovian conditioning: Facilitation or impairment of acquisition as a function of individual differences in responsiveness to the CS. Journal of Experimental Psychology: Animal Behavior Processes, 1977, 3, 357-367.

Gormezano, I. Classical conditioning. In J. B. Sidowski (Ed.), Experimental methods and instrumentation in psychology. New York: McGraw-Hill, 1966.

Hintzman, D. L. Simpson's paradox and the analysis of memory retrieval. Psychology Review, 1980, 87, 398-410.

Kamin, L. J. Attention-like processes in classical conditioning. In M. R. Jones (Ed.), Miami Symposium on the Prediction of Behavior. Miami: University of Miami Press, 1968.

Kamin, L. J. Predictability, surprise, attention and conditioning. In R. M. Church B. Campbell (Eds.), Punishment and aversive behavior. New York: Appleton-Century-Crofts, 1969.

Kettlewell, N. M., O'Connell, M. F., \& Berger, L. H. Bilateral nictitating membrane conditioning in rabbits under asymmetrical levels of cutaneous afferent activity. Physiology \& Behavior, 1974, 13, 27-33.

Kimmel, H., \& ReYnolds, T. W. On the locus of extinctive inhibition. Acta Neurobiologica Experimentalis, 1971, 31, 227-236.

LAsole, J., \& Bindra, D. An interpretation of autoshaping and related phenomena in terms of stimulus-incentive contingencies alone. Canadian Journal of Psychology, 1976, 30, 157-173.

LeYlaND, C. F., \& MACKintosh, N. J. Blocking of first- and second-order autoshaping in pigeons. Animal Learning $B e$ havior, 1978, 6, 391-394.

MackinTosh, N. J. Cognitive or associative theories of conditioning: Implications of an analysis of blocking. In S. H. Hulse, H. Fowler, \& W. K. Honig (Eds.), Cognitive processes in animal behavior. Hillsdale, N. J: Erlbaum, 1978.

Mackintosh, N. J., Bygrave, D. J., \& Picton, B. M. B. Locus of the effect of a surprising reinforcer on the attenuation of blocking. Quarterly Journal of Experimental Psychology, 1977, 29, 327-336.

Mackintosh, N. J., \& Turner, C. Blocking as a function of novelty of CS and predictability of UCS. Quarterly Journal of Experimental Psychology, 1971, 23, 359-366.

Marchant, H. G., \& Moore, J. W. Blocking of the rabbit's nictitating membrane response in Kamin's two-stage paradigm. Journal of Experimental Psychology, 1973, 101, 155-158.

Mis, F. W., \& Moons, J. W. Effects of preacquisition UCS exposure on classical conditioning of the rabbit's nictitating membrane response. Learning and Motivation, 1973, 4, 108-114.

Moone, J. W., \& Stckney, K. J. Formation of attentionalassociative networks in real time: Role of the hippocampus and implications for conditioning. Physiological Psychology, 1980, 8, 207-217.

Penrce, J. M., \& Hall, G. A model for Pavlovian learning: Variations in the effectiveness of conditioned stimuli but not of unconditioned stimuli. Psychological Review, 1980, 87, 532-552.

Penrce, J. M., Montgomery, A., \& Dickingon, A. Contralateral transfer of inhibitory and excitatory eyelid conditioning in the rabbit. Quarterly Journal of Experimental Psychology, 1981, 33, 45-61.

Prokasy, W. F., \& Gormezano, I. The effects of US omission in classical aversive and appetitive conditioning of rabbits. Animal Learning \& Behavior, 1979, 7, 80-88.

REscorla, R. A. Conditioned inhibition of fear. In N. J. Mackintosh \& W. K. Honig (Eds.), Fundamental issues in associative learning. Halifax, Nova Scotia: Dalhousie University Press, 1969.

Resconla, R. A. Pavlovian second-order conditioning: Studies in associative learning. Hillsdale, N. J: Erlbaum, 1980. 
Rescorla, R. A., \& Wagner, A. R. A theory of Pavlovian conditioning: Variations in the effectiveness of reinforcement and non-reinforcement. In A. N. Black \& W. F. Prokasy (Eds.), Classical conditioning II: Current theory and research. New York: Appleton-Century-Crofts, 1972.

Roze воOм, W. W. "What is learned?"-An empirical enigma. Psychological Review, 1958, 65, 22-23.

Salafia, W. R., Daston, A. P., Bartogiak, R. S., Hurley, J., \& Martino, L. J. Classical nictitating membrane conditioning in rabbit as a function of US locus. Journal of Comparative and Physiological Psychology, 1974, 86, 628-636.

Suboski, M. C., Dilollo, V., \& Gormezano, I. Effects of unpaired preacquisition exposure of the CS and the UCS on classical conditioning of the nictitating membrane response of the albino rabbit. Psychological Reports, 1964, 15, 571-576.
TERRY, W. S. Short-term memory for "surprising" versus "expected" unconditioned stimuli in Pavlovian conditioning. Journal of Experimental Psychology: Animal Behavior Processes, 1976, 1, 122-133.

WAGNer, A. R. SOP: A model of automatic memory processing in animal behavior. In N. E. Spear \& R. R. Miller (Eds.), Information processing in animals: Memory mechanisms. Hillsdale, N.J: Erlbaum, 1981.

Wagner, A. R., Mazur, J. E., Donegan, N., \& Prautz, P. L. Evaluation of blocking and conditioned inhibition to a $\mathrm{CS}$ signalling a decrease in US intensity. Journal of Experimental Psychology: Animal Behavior Processes, 1980, 6, 376-397.

(Manuscript received March 2, 1982;

revision accepted for publication September 14, 1982.) 